\title{
The tensile and shear failure behavior dependence on chain length and temperature in amorphous polymers
}

\author{
Junhua Zhao*, Timon Rabczuk ${ }^{*}$ \\ Institute of Structural Mechanics, Bauhaus-University Weimar, 99423 Weimar, Germany \\ *Corresponding author: junhua.zhao@uni-weimar.de; timon.rabczuk@uni-weimar.de
}

\begin{abstract}
The tensile and shear failure behavior dependence on chain length and temperature in amorphous polymers are scrutinized using molecular dynamics simulations. A wide range chain length of alkane is tested under tension and shear with various temperatures. We find that the broken rate (the broken bond number to all polymer chain number ratios) under tension and shear increases with increasing chain length and temperature. For a given chain length and temperature, the broken rates under shear are always higher than those under tension at a same large strain. For a given chain length, the tensile and shear stresses decrease with increasing temperature. We propose three typical fracture mechanisms to effectively elucidate the ductile fracture response based on the predominance of chain scission process.
\end{abstract}

Keywords Failure, Chain length, Linear polymers, Molecular dynamics.

\section{Introduction}

Amorphous polymers are one of the most fundamental polymer molecular shapes that have widely been investigated by many researchers due to the important physical and chemical properties [1,2]. Glass forming polymers $\left(T<T_{\mathrm{g}}, T_{\mathrm{g}}\right.$ is the glass-transition temperature) are of great industrial importance and scientific interest. Their unique mechanical properties arise from the connectivity and random-walk-like structure of the constituent chains [3]. At very small strains, the response is elastic. At slightly larger strains, yielding occurs when intermolecular barriers to segmental rearrangements are overcome. Following yield, the material may exhibit strain softening, a reduction in stress to a level corresponding to plastic flow. At higher strains, the stress increases as the chain molecules orient, in a process known as strain hardening. Strain hardening suppresses strain localization (crazing, necking, shear banding) and is critical in determining material properties such as toughness and wear resistance [4,5]. In the other hand, the yield point of the polymers disappears after $T>T_{\mathrm{g}}$. Recently, we have found that the chain length (CL) and temperature have a large effect on the thermomechanical properties of linear polymers [6-8] based on united-atom (UA) and coarse-grained (CG) molecular dynamics (MD) simulations. Since the UA and CG potentials limitations, the effect of the CL and temperature on the failure behavior is not understood well yet. Especially, the failure behavior under shear has been scarcely reported in previous work. Therefore, understanding the molecular origins of macroscopic fracture behavior such as fracture energy is a fundamental scientific challenge [5,9].

In this paper, the tensile and shear failure behavior dependence on CL and temperature in linear polymers are scrutinized using MD simulations. A wide range chain length of alkane is tested under tension and shear with various temperatures. The fracture mechanism is proposed based on the detailed analysis of the fracture response.

\section{Simulations details}


The bulk structure of linear polymers can be modeled using the semicrystalline lattice method [10], which utilizes the face-centered cubic $(f c c)$ diamond structure as a template to carbon backbones of entangled polymers. The detailed process was detailedly described in our previous work [6]. The total number of beads in the initial structure is kept constant 180000 from CL=9 to $\mathrm{CL}=1200$, in which the number of chains changes accordingly from 20000 (CL=9) to 150 $(C L=1200)$. Since our aim does not simulate a specific polymer, we use a bead-spring polymer model derived from the one suggested by Kremer and Grest [11]. A finitely extendable nonlinear elastic (FENE) backbond potential is applied along the polymer chain

$$
U(r)=-\frac{k}{2} R_{0}^{2} \ln \left[1-\left(\frac{r}{R_{0}}\right)^{2}\right],
$$

where $k=30$ and $R_{0}=1.5$ to guarantee a certain stiffness of the bonds while avoiding high frequency modes (which would require a rather small time step for the integration) and chain crossing [12]. The beads interact through a truncated Lennard-Jones (LJ) potential of the form

$$
\left\{\begin{array}{c}
U_{L J}(r)=4 \in\left[\left(\frac{\sigma}{r}\right)^{12}-\left(\frac{\sigma}{r}\right)^{6}-\left(\frac{\sigma}{r_{c}}\right)^{12}+\left(\frac{\sigma}{r_{c}}\right)^{6}\right], r<r_{c}=1.5 \sigma \\
U_{L J}(r)=0, r>r_{c}
\end{array},\right.
$$

where $\varepsilon$ and $\sigma$ are the characteristic energy and distance parameters that define the shape of the energy distance curve, while $r_{\mathrm{c}}$ is the cutoff distance for the potential. The LJ potential provides a smooth transition to zero values at the cutoff distance. In this work, we adopt the reduced units formalism and all physical quantities are expressed as multiples of $m$ (bead mass), $\varepsilon, \sigma$ and $k_{\mathrm{b}}$ (Boltzmann constant) while these parameters are set equal to one in our computation [13]. The bond is broken as $r>1.15 \sigma$ and the corresponding interaction is shut off, while a non-bonded LJ interaction is introduced between the two beads.

Each generated initial three-dimensional structure is annealed for $1 \times 10^{6}$ steps until the pressure and energy of the system is stable, keeping both the temperature $T=1.3 \varepsilon / k_{\mathrm{b}}$ and the pressure $P=1$ (the time step $d t=0.002$ ) in the $N P T$ ensemble controlled by the Nose-Hoover's thermostat [14]. Then, the system is cooled down to be the given temperature by the same NPT ensemble and the density of the system is monitored while cooling step-wise at an effective rate of $1 /\left(1 \times 10^{6}\right.$ steps $)$. The system is then kept at the constant temperature (the given temperature) for $1 \times 10^{6}$ steps in the same NPT ensemble. The obtained structures are subjected to the uniaxial tension and compression and performed to obtain stress-strain response with different CL and temperature in the non-equilibrium MD simulations [15]. Periodic boundary conditions are applied in all directions. All the MD simulations have been performed using LAMMPS software [16].

\section{Results and discussion}

\subsection{Failure behavior under uniaxial tension}

Fig. 1 shows the stress-strain curves with different CL for two different temperature $T=0.1$ and $T=0.3$. The typical process (elastic, yielding, softening and hardening) is displayed in Fig. 1, while the hardening process is not evident when CL $\leq 18$ and the difference among the nonlinear parts of the stress-strain curves is also not large when CL $\leq 144$. The main reason is that the density and entanglement of short CL is lower than those of longer chain, which results in that 
the bond number per unit volume in short CL is lower than those in longer CL. So the required external work for the shorter CL is lower than that for the longer CL. That is to say, the stiffness of the shorter CL is lower than that of longer CL. Since the density almost tends to a constant when $C L \geq 140$ [6], the effect is not evident in the range of $C L \geq 140$. Fig. 2 shows bond broken rate (the number of broken bond / the number of total chains) with different tensile strain for different CL and two temperatures $T=0.1$ and $T=0.3$, in which the broke rate increases with increasing CL.
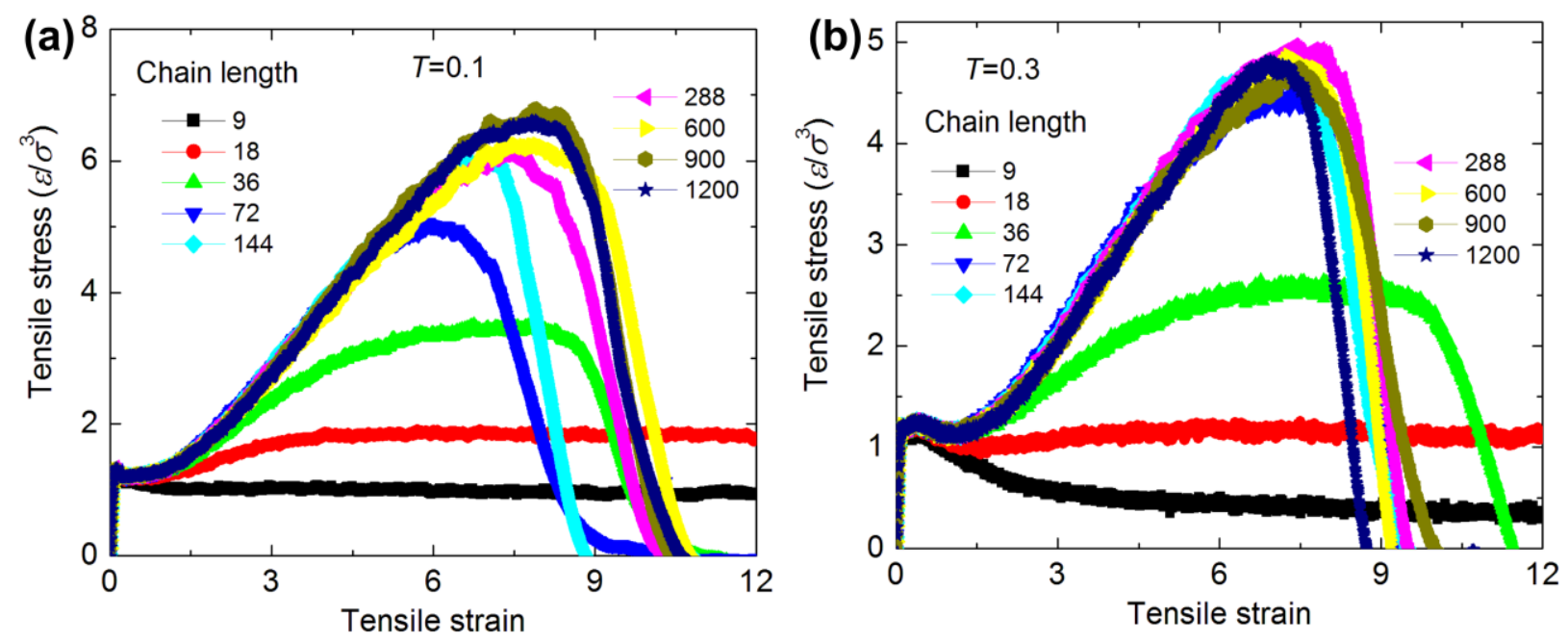

Fig. 1 The tensile stress-strain curves with different chain length for two different temperatures. (a) $T=0.1$; (b) $T=0.3$.
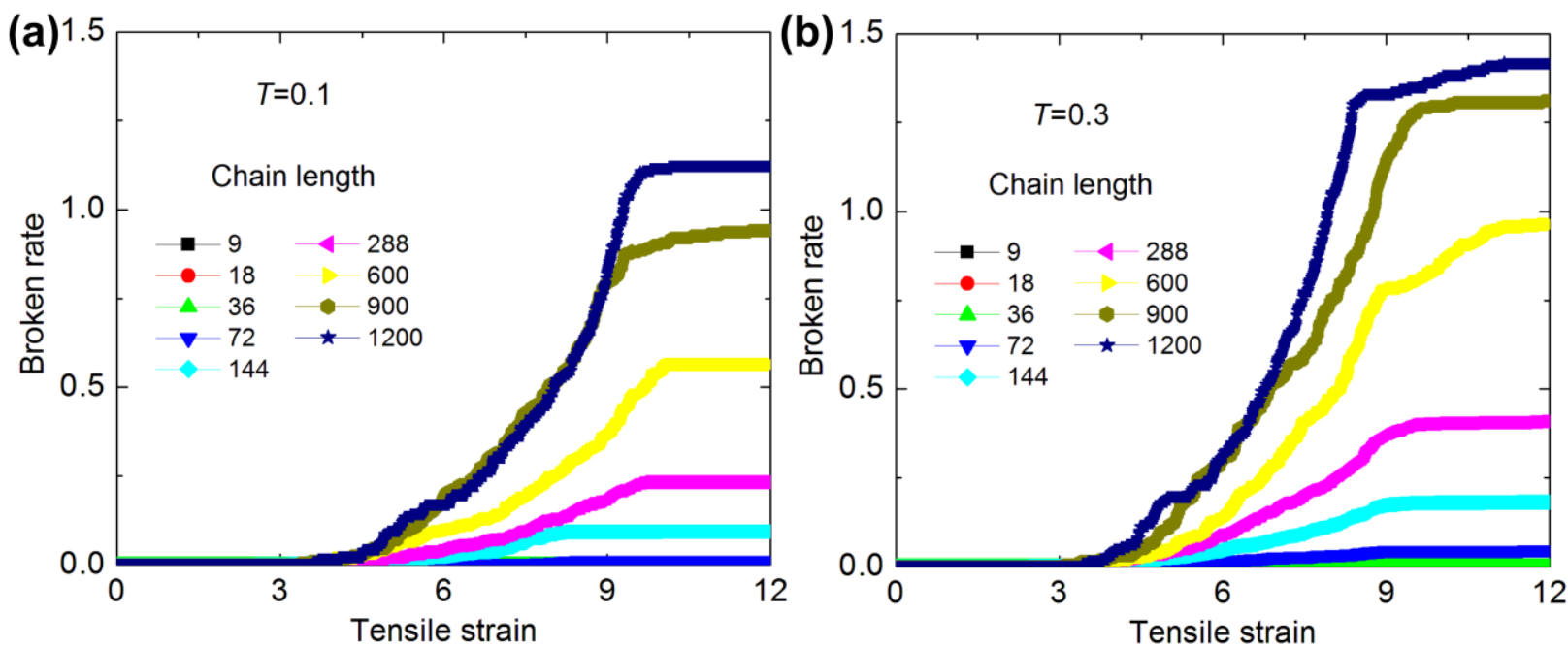

Fig. 2 The broken rate-strain curves with different chain length for two different temperatures. (a) $T=0.1$; (b) $T=0.3$.

We also find that the first broken bond is occurred at strain $>3$ and the stress is still hardening with increasing strain by comparison with Fig. 1 and Fig. 2. After strain $>9$, the stress decreases and the number of the total broken bonds tends to a constant. Fig. 3 shows the stress-strain curves and broken rate for $\mathrm{CL}=288$ with different temperature. The stress decreases and the 
broken rate increases with increasing temperature for same strain. For high temperature ( $T=0.5$ and 0.7), the number of broken bond always increases with increasing strain even if the strain is higher than 12. The possible reason is that the temperature is higher than the glass-transition temperature $T_{\mathrm{g}}=0.35$ (here) when $T=0.5$ and 0.7 , in which the creep and high-elastic property is more evident in the high temperature and the yield point disappears. To interpret these MD simulations and obtain insight into the difference in fracture behavior of different CL and temperature, we plot the atomic strain distribution in the deformed configuration of the polymer models in Fig. 4 and Fig. 5. The ultimate fracture strain increases with increasing temperature in Fig. 4, which gives a good explanation of the above analysis. In Fig. 5, no void or atomic strain concentration for $\mathrm{CL}=18$ can be found even at strain=12, which validates no broken bond in Fig. 2a. The ultimate fracture strain almost increases with increasing CL when CL $\geq 72$, which is also validated from Fig. 2a.
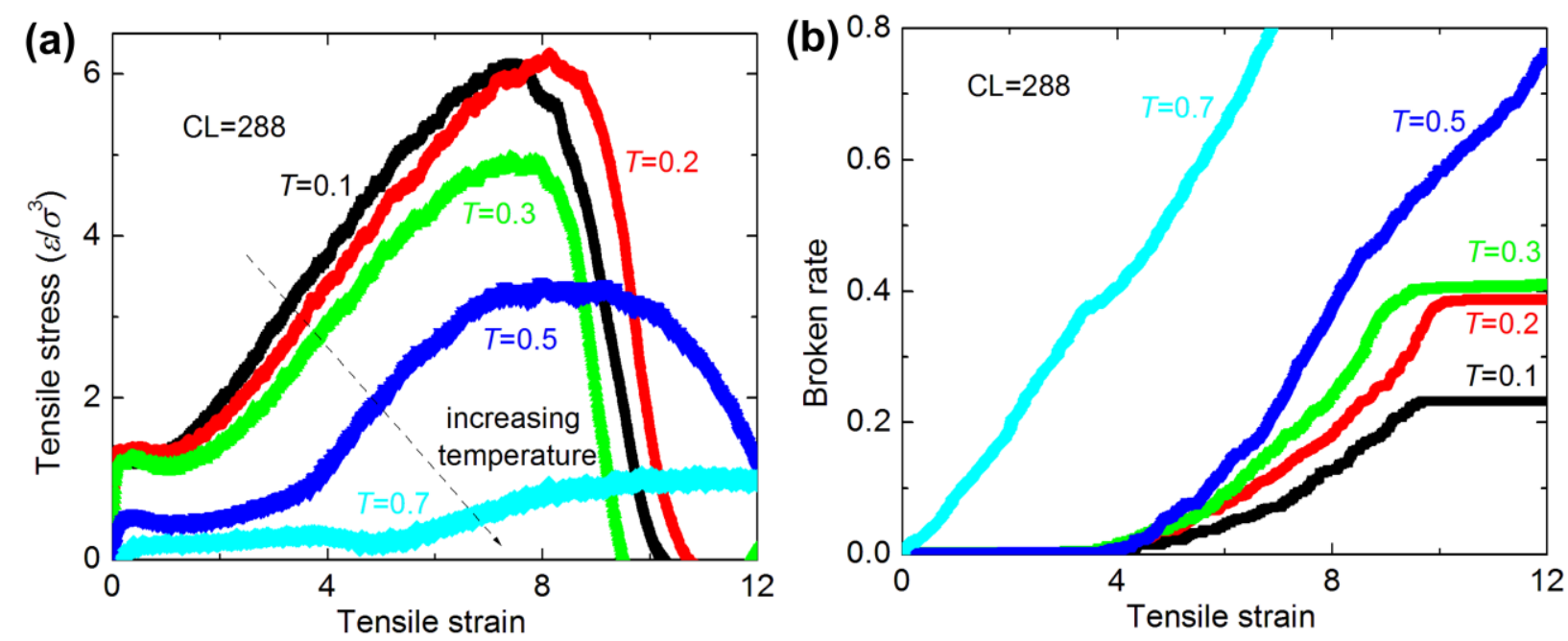

Fig. 3 The tensile stress-strain and broken rate-strain curves with different temperature for $C L=288$. (a) Stress-strain curves; (b) Broken rate-strain curves.

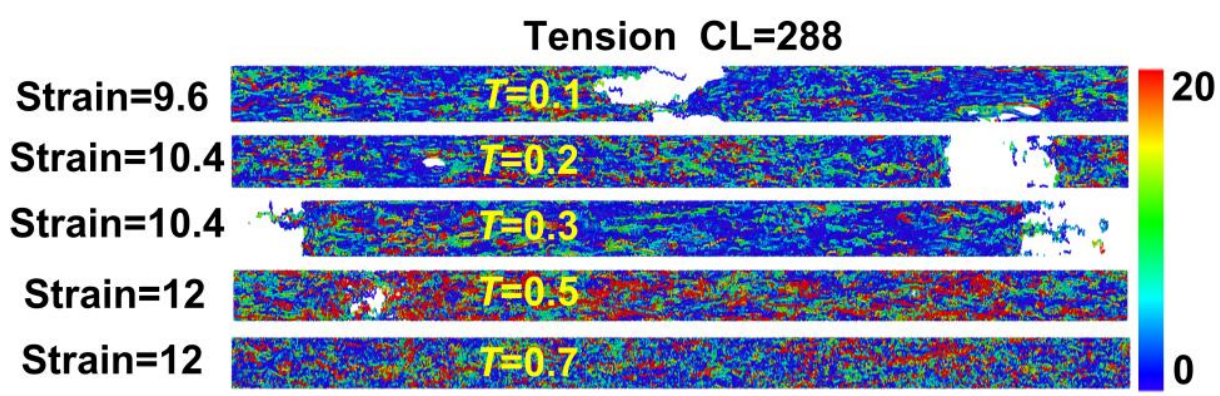

Fig. 4 The atomic strain (along the tensile direction) distribution for $\mathrm{CL}=288$ at different temperature. 
Tension $T=0.1$

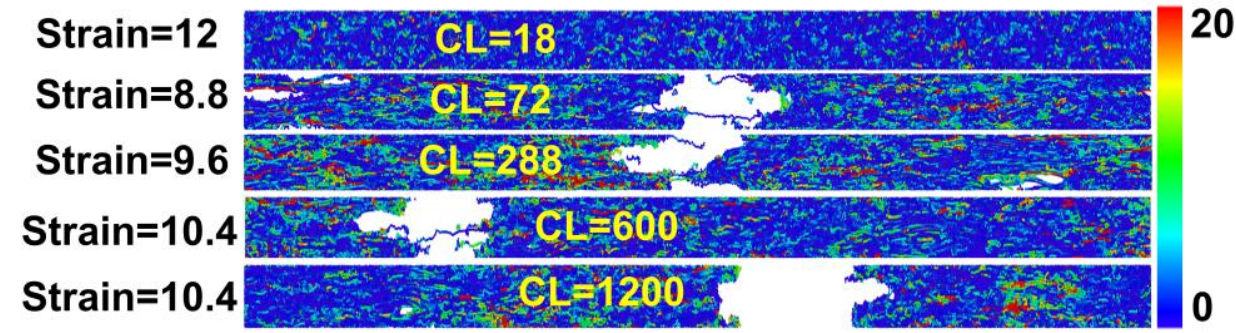

Fig. 5 The atomic strain (along the tensile direction) distribution for different CL at $T=0.1$.

Three typical mechanisms of the fracture behavior under uniaxial tension are plotted in Fig. 6. Fig. 6a represents the covalent bond broken phenomenon, which requires very high external loading (about $15000 \mathrm{MPa}$ for linear polymers). Fig. 6b represents the polymer chain slipping with each other (about $5000 \mathrm{MPa}$ ). Fig. 6c represents the separation between any two polymer chains, which is mainly dominated by van der Waals interactions (about $100 \mathrm{MPa}$ ). For short CL, the fracture behavior is mainly determined by the chain slipping and separation. For longer CL, the fracture behavior is dominated by the competition between the chain slipping and bond broken mechanisms, in which the bond broken mechanism increases with increasing CL since the entanglement density increases with increasing CL. For very long CL, the bond broken mechanism mainly dominates the fracture behavior. The three mechanisms provide a good explanation of the MD results from Fig. 1 to Fig. 5.

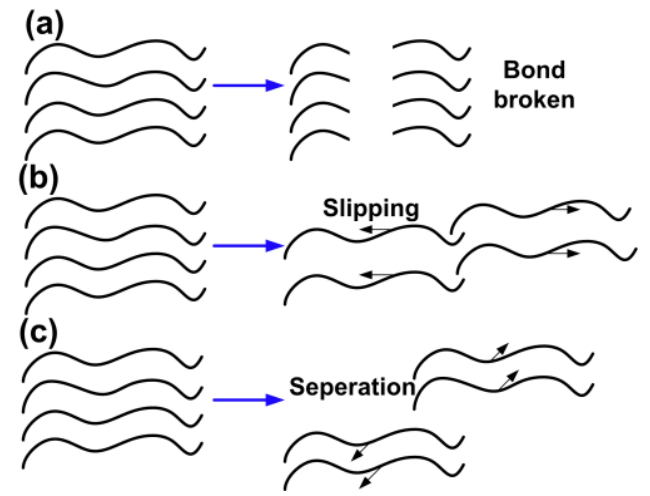

Fig. 6 Three typical mechanisms of microstructural evolution under uniaxial tension in linear polymers.

\subsection{Failure behavior under shear}

Fig. 7 shows the shear stress-strain curves with different CL for two different temperatures $T=0.1$ and $T=0.3$. The typical process (elastic, yielding, softening and hardening) is also displayed in Fig. 7. Unlike tension, the hardening process is also evident even for very short CL. The stress strength (the peak point of the stress) and strain strength (the strain at the stress strength point) both increase with increasing CL when $C L \leq 144$. The possible reason is that the polymer chains mainly keep the slipping process even in the hardening stage under shear, while the polymer chains are mainly stretching process after elastic stage under tension. 

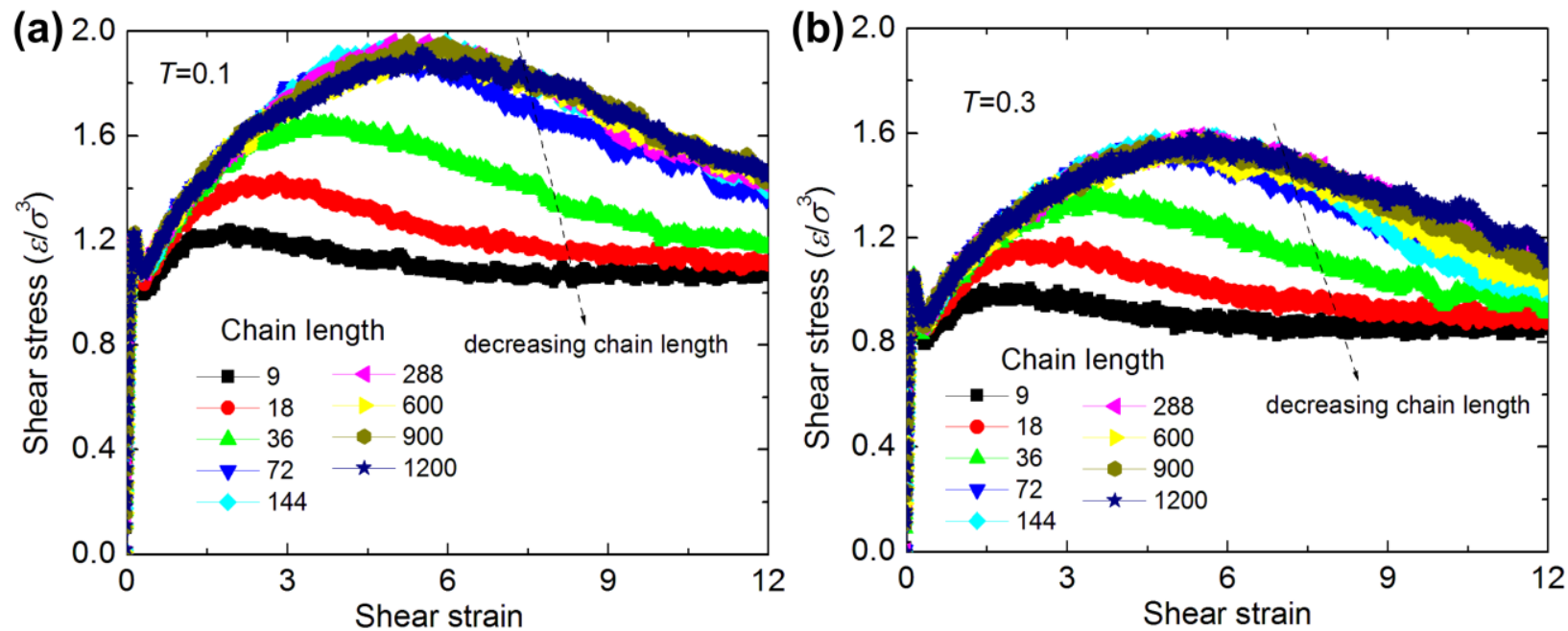

Fig. 7 The shear stress-strain curves with different chain length for two different temperatures. (a) $T=0.1$; (b) $T=0.3$.

Fig. 8 shows the corresponding broken rate with different CL for two different temperatures of Fig. 7. Similar to tension, the shear broken rate also increases with increasing CL. Unlike tension, the broke rate always increases with increasing shear strain even strain $>12$. Fig. 9 shows the ratio of tensile broken rate to shear broken rate with different strain. The ratio is always smaller than 1 . In other words, for a given chain length and temperature, the broken rates under shear are always higher than those under tension for a same large strain. The possible reason is that the slipping and separation process mainly dominate the forward stage of the fracture behavior under shear, while the coupling slipping and broken mechanisms determine the fracture behavior under uniaxial tension. The $r_{\mathrm{c}}=1.5$ of LJ potential is higher than the broken distance of $r_{\text {broken }}=1.15$ (see section 2), which leads to the higher ultimate fracture strain (that is, the structure is broken as two parts) under shear.
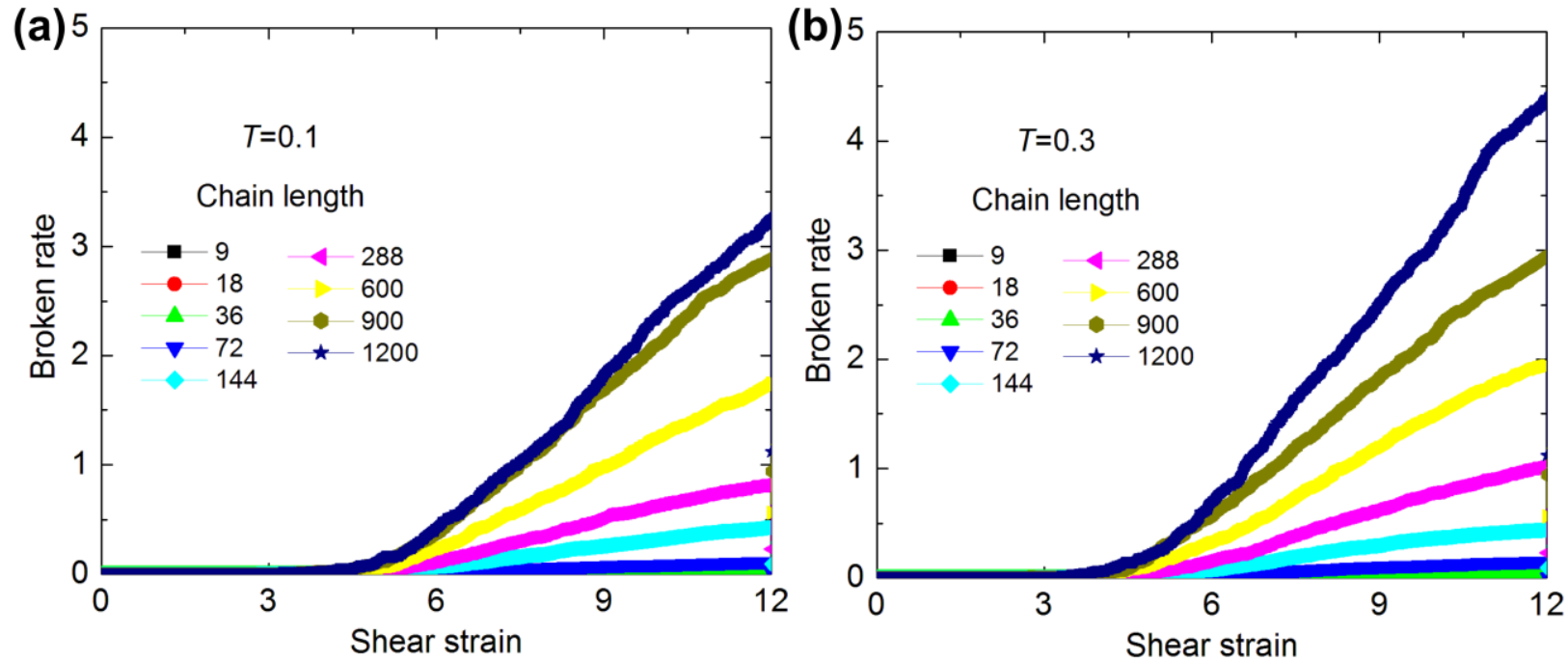

Fig. 8 The shear broken rate-strain curves with different chain length for two different temperature. (a) $T=0.1$; (b) $T=0.3$. 

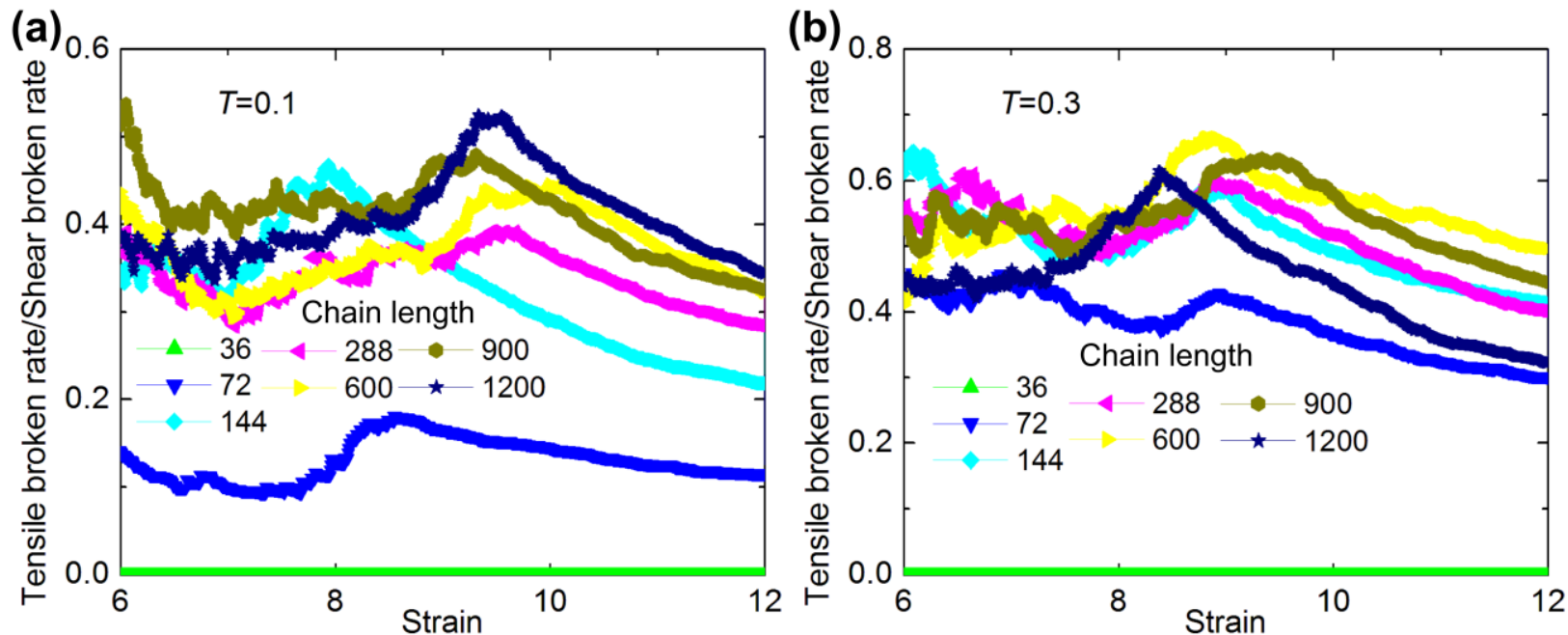

Fig. 9 The tensile broken rate/shear broken rate with different chain length for different temperature. (a) $T=0.1$; (b) $T=0.3$.
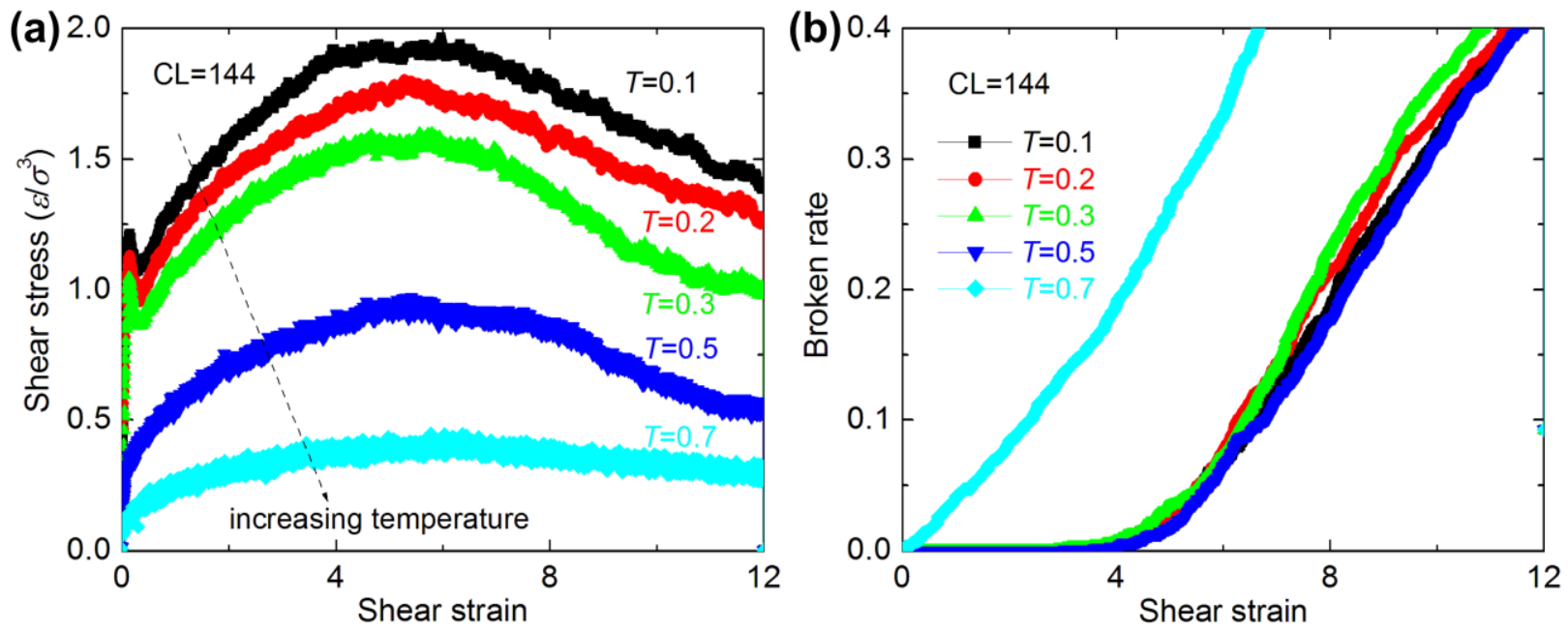

Fig. 10 The shear stress-strain and broken rate-strain curves with different temperature for $C L=144$. (a) Stress-strain curves; (b) Broken rate-strain curves.

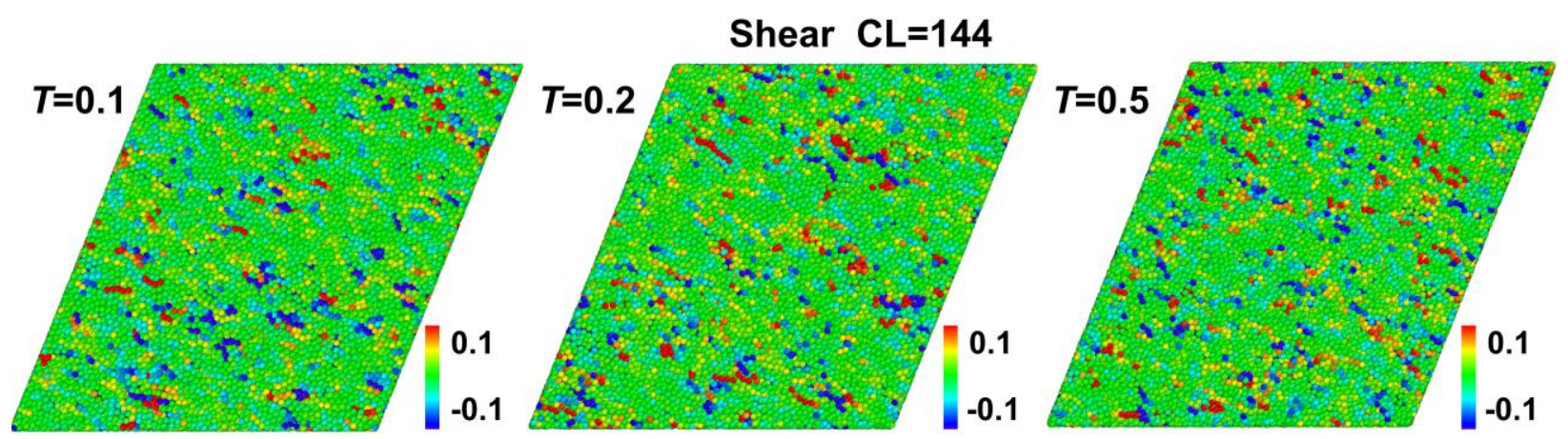

Fig. 11 The atomic strain (along the shear direction) distribution for $\mathrm{CL}=144$ at different temperature under shear strain $=58 \%$. 
Fig. 10 shows the shear stress-strain curves and broken rate for $\mathrm{CL}=144$ with different temperature. The shear stress decreases with increasing temperature for same strain. For high temperature ( $T=0.5$ and 0.7 ), no evident yield point can be also seen in Fig. 10a, which is same with tension. The difference of shear broken rate for different temperature is not large as $T<0.7$. To further understand the response, we plot the atomic strain (along the shear direction) distribution under shear strain (58\%) for different temperatures and CL in the deformed configuration of the polymer models in Fig. 11 and Fig. 12. For $\mathrm{CL}=144$ under same shear strain, the difference of the atomic strain distribution with different temperature is not evident in Fig. 11. For $T=0.1$, the atomic strain concentration increases with increasing CL in Fig. 12. The possible reason is that more entanglements are in large $\mathrm{CL}$, which leads to the atomic stress concentration at these positions.

From above analysis, the ultimate fracture strain increases with increasing temperature under uniaxial tension. Under uniaxial tension, the fracture behavior is mainly determined by the chain slipping and separation for short CL. For longer CL, the fracture behavior is dominated by the competition between the chain slipping and bond broken mechanisms, in which the bond broken mechanism increases with increasing CL since the entanglement density increases with increasing CL. For very long CL, the bond broken mechanism mainly dominates the fracture behavior. Under shear, the atomic strain concentration increases with increasing CL for a given temperature. The shear broken rate is always higher than that under tension for same CL in the same large strain. The possible reason is that the slipping and separation process mainly dominate the forward stage of the fracture behavior under shear, while the coupling slipping and broken mechanisms determine the fracture behavior under uniaxial tension. The $r_{\mathrm{c}}=1.5$ of $\mathrm{LJ}$ potential is higher than the broken distance of $r_{\text {broken }}=1.15$ (see section 2), which leads to the higher ultimate fracture strain (that is, the structure is broken as two parts) under shear.

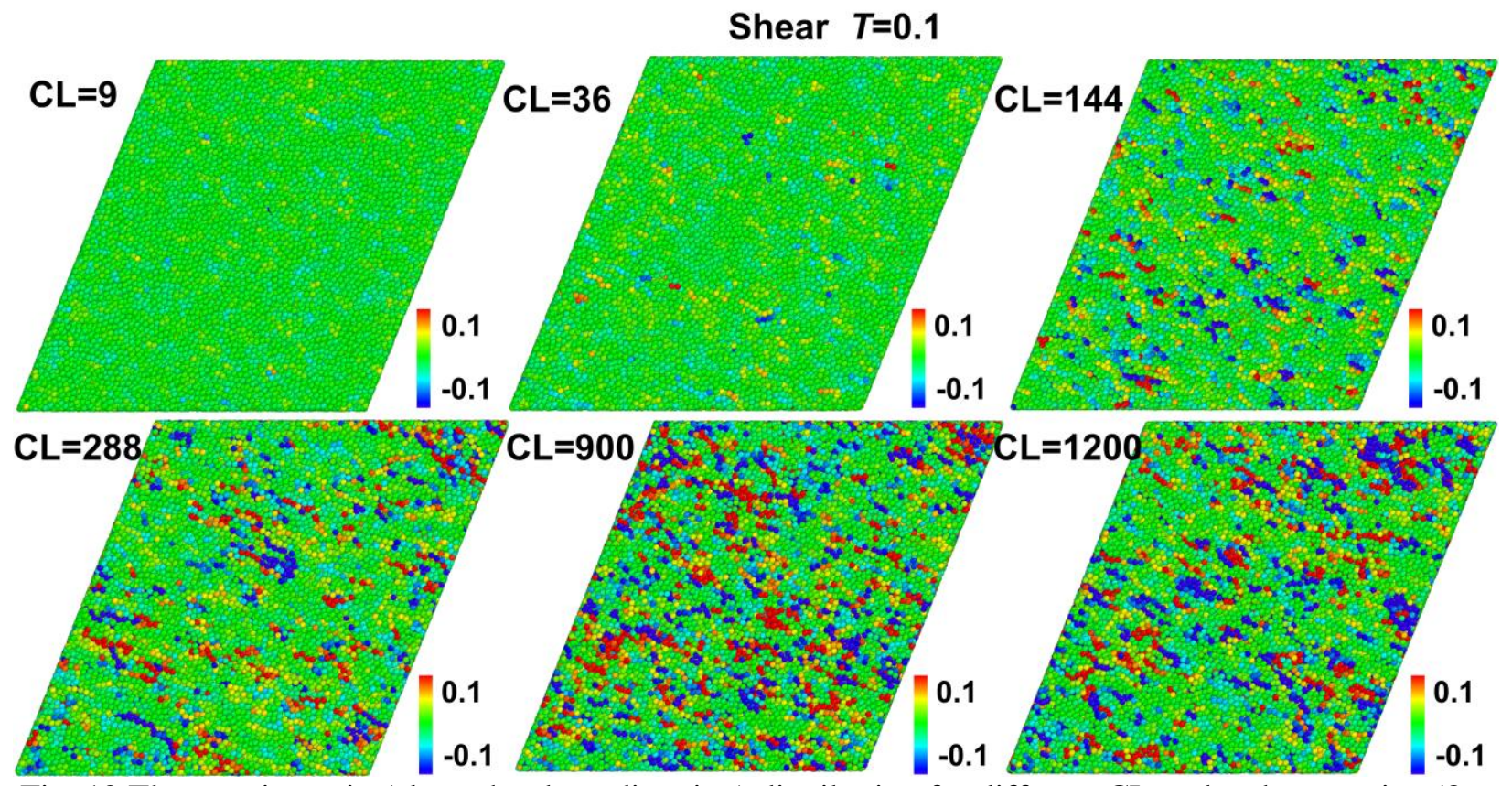

Fig. 12 The atomic strain (along the shear direction) distribution for different CL under shear strain=58\% at $T=0.1$. 


\section{Concluding remarks}

In summary, the tensile and shear failure behavior dependence on chain length and temperature in linear polymers are investigated using molecular dynamics simulations. A wide range chain length of alkane is tested under tension and shear with various temperatures. We find that the broken rate (the broken bond number to all polymer chain number ratios) under tension and shear increases with increasing chain length and temperature. For a given chain length and temperature, the broken rates under shear are always higher than those under tension at a same large strain. For a given chain length, the tensile and shear stresses decrease with increasing temperature. Under uniaxial tension, the fracture behavior is mainly determined by the chain slipping and separation for short CL. For longer CL, the fracture behavior is dominated by the competition between the chain slipping and bond broken mechanisms, in which the bond broken mechanism increases with increasing CL. For very long CL, the bond broken mechanism mainly dominates the fracture behavior. The slipping and separation process mainly dominate the forward stage of the fracture behavior under shear. The $r_{\mathrm{c}}=1.5$ of $\mathrm{LJ}$ potential is higher than $r_{\mathrm{F}}=1.15$ bond broken, which leads to the higher ultimate fracture strain under shear than that under uniaxial tension.

\section{Acknowledgements}

We gratefully acknowledge support by the Germany Science Foundation and and IRSES-MULTIFRAC project.

\section{References}

[1] R.H. Boyd, R.H. Gee, Y. Jin, Conformational dynamics in bulk polyethylene: a molecular dynamics simulation study. J Chem Phys, 101 (1994) 788-797.

[2] P.V.K. Pant, J. Han, G.D. Smith, R.H. Boyd, A molecular dynamics simulation of polyethylene. J Chem Phys, 99 (1993) 597-604.

[3] J.E. Shepherd, Multiscale modeling of the deformation of semi-crystalline polymers. Ph. D thesis. Georgia Institute of Technology, USA, 2006.

[4] R.S. Hoy, M.O. Robbins, Strain hardening of polymer glasses: Entanglements, energetics, and plasticity. Phys Rew E, 77(2008) 031801.

[5] R.S. Hoy, M.O. Robbins, Strain hardening in polymer glasses: limitations of network models. Phys. Rev. Lett. 99 (2007) 117801.

[6] J. Zhao, S. Nagao, Z.L. Zhang, Thermo-mechanical properties dependence on chain length in bulk polyethylene: Coarse-grained molecular dynamics simulations, J Mater Res, 25 (2010) 537-544.

[7] J.H. Zhao, S. Nagao, Z.L. Zhang, H. Kristiansen, Coarse-grained molecular dynamics simulations on size effect of glassy polyethylene particles. J Nanosci Nanotech, 10 (2010) 7340-7342.

[8] J. Zhao, J.W. Jiang, N. Wei, Y. Zhang, T. Rabczuk, Thermal conductivity dependence on chain length in amorphous polymers. Appl Phys Lett, under review, 2013.

[9] H.G.H. van Melick, L.E. Govaert, H.E.H. Meijer, On the origin of strain hardening in glassy polymers. Polymer, 44 (2003) 2493-2502.

[10] J.L. Faulon, Stochastic generator of chemical structure. (4) Building polymeric systems with specified properties. J Comput Chem, 22 (2001) 580-590. 
[11] K. Kremer, G.S. Grest, Dynamics of entangled linear polymer melts: A molecular dynamics simulation. J Chem Phys, 92 (1990) 5057-5086.

[12] C. Bennemann, W. Paul, K. Binder, Molecular-dynamics simulations of the thermal glass transition in polymer melts: $\alpha$-relaxation behavior. Phys Rev E, 57 (1998) 843-851.

[13] M. Panico, S. Narayanan, L.C. Brinson. Simulations of tensile failure in glassy polymers: effect of cross-link density. Modelling Simul Mater Sci Eng, 18 (2010) 055005.

[14] S. Nose, A unified formulation of the constant temperature molecular dynamics methods. J Chem Phys, 81 (1984) 511-519.

[15] F.M. Capaldi, M.C. Boyce, G.C. Rutledge, Molecular response of a glassy polymer to active deformation. Polymer, 45 (2004) 1391-1399.

[16] S. Plimpton, Fast parallel algorithms for short-range molecular dynamics. J Comput Phys, 117 (1995) 1-19. 